\title{
Gustav Klimt (1862-1918) y su cuadro sobre la medicina
}

\author{
BÁRBARA C. FINN, JULIO E. BRUETMAN, PABLO YOUNG
}

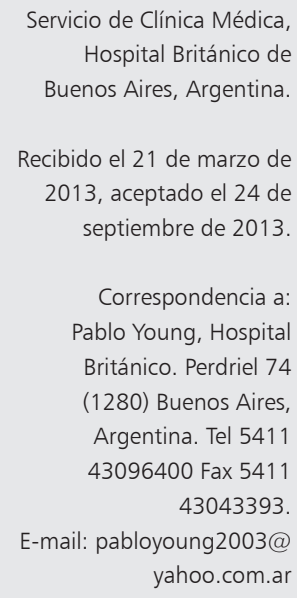

\section{The portrait of medicine and Gustav Klimt (1862-1918)}

Gustav Klimt was an Austrian Symbolist painter and one of the most important representatives of the modernist movement of the Viennese Secession. The most notable works carried out at his golden age were the "Portrait of Adele Bloch-Bauer I" and "The kiss". In 1901 he paints "Medicine" where he returns to the entanglement of floating bodies -the lifetime- among which appears the skeleton of death; a female figure stands out from the column to represent freedom from pain. The figure of $\mathrm{Hy}$ geia or Hygieia, daughter of Aesculapius, who personifies medicine, is shown in the foreground. In 1911, thanks to "The life and death", a picture related to medicine, he was awarded the first prize at the Universal Exhibition of Rome. A number of his artworks were confiscated by the Nazi dictatorship. During the advance of enemy troops, and fearing that these works would become spoils of war, it was decided to burn the castle where they remained confiscated. Therefore, countless artworks were lost. The progressive development of symbolic or abstract motifs already emphasized the freedom of spirit that permeated all the avant-garde of early twentieth century.

(Rev Med Chile 2013; 141: 1584-1588)

Key words: Anatomy; Portraits; History of Medicine.

\section{$\underline{\text { Klimt, el pintor del imperio austrohúngaro }}$}

G ustav Klimt (1862-1918) (Figura 1) fue un pintor simbolista austríaco y uno de los más conspicuos representantes del movimiento modernista de la "Secesión vienesa"1-4.

Nació en Baumgarten, cerca de Viena. Vivió en relativa pobreza la mayor parte de su infancia. Gracias a su talento, en 1876, con catorce años, recibió una beca para estudiar en la Escuela de Artes y Oficios de Viena, donde se formaría hasta 1883 como pintor y decorador de interiores. Gustav, su hermano menor Ernst (1864-1892) y su amigo Franz Matsch (1861-1942) eran tenidos en alta estima por sus profesores, quienes a menudo los recomendaban para el trabajo remunerado fuera de la escuela.

El imperio de los Habsburgo había entrado en su fase final y su capital, Viena, uno de los centros culturales y científicos florecientes de Europa, se encontraba bajo un régimen liberal experimentando un período de expansión industrial. La antigua ciudad de Viena contaba con una muralla perimetral construida en el siglo XIII. En 1857 el emperador Francisco José I (1830-1916) ordenó demoler la muralla y construir en su lugar un gran boulevard rodeado de opulentos edificios públicos y privados. Este boulevard, llamado Ringstrasse, o "calle anillo", circunda la ciudad y los edificios previstos pretendían ser una muestra de la grandeza y la gloria imperiales.

Los tres jóvenes artistas participaron en el ambicioso proyecto, decorando la gran escalinata del nuevo teatro -el Burgtheater-desde 1888 hasta 1890 y, en 1891, el gran vestíbulo del Museo de Historia del Arte. En 1888, Klimt recibió la Orden de Oro al Mérito de manos del emperador Francisco José I por su trabajo en los murales del salón de actos del Burgtheater, donde están representados cerca de 200 personalidades del teatro vienés. 
Estas primeras obras, aunque creadas en el estilo convencional de la época, ya anunciaban su talento versátil. El encargo para realizar las pinturas de la Universidad de Viena, como veremos posteriormente, era una consecuencia lógica.

A principios de la década de 1890, conoció a Emilie Flöge (1874-1952), quien soportó las constantes aventuras amorosas del artista y se convertiría en su compañera hasta el final de su vida. Tuvieron al menos catorce hijos.

Para el año 1892 su padre y hermano Ernst murieron, y ya estaba en condiciones de soportar la carga económica de sus parientes. La tragedia familiar pesó también en su expresión artística y marcó el inicio de la definición de su estilo personal ${ }^{1}$.

Klimt se transformó en un pintor simbolista y uno de los más conspicuos representantes del movimiento de la "Secesión vienesa", parte de lo que hoy llamamos "modernismo". Uno de los primeros grupos que se rebelaron contra las normas académicas y tradicionales ("secesionistas") sería el grupo literario "Joven Viena”, pero la verdadera "Secesión” se gestó en la Casa de los Artistas, única asociación de artistas de Viena que organizaba temporalmente exposiciones para mostrar las creaciones de sus miembros, exposiciones que contaban con un jurado que seleccionaba a los artistas participantes. Los miembros antiguos no permitían incorporar en las muestras obras de artistas que tuvieran una visión diferente y, por supuesto, no se permitía la exhibición de obras realizadas por artistas extranjeros, canalizando las muestras de manera casi exclusivamente comercial ${ }^{2}$.

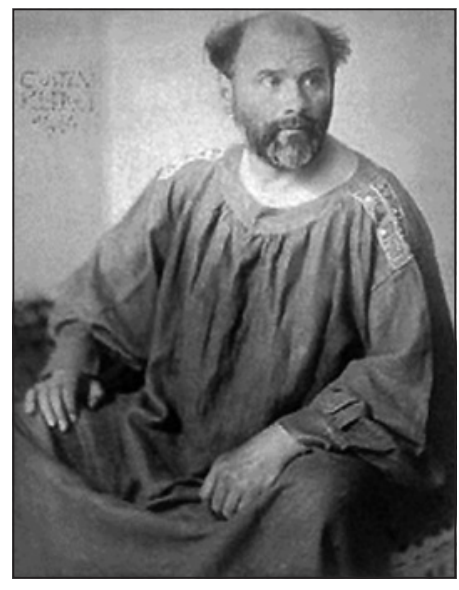

Figura 1. Gustav Klimt. En: http://artofgustavklimt.blogspot.com.ar/2008/10/ art-of-gustav-klimt. html; consultado 10/3/2013.
En 1897 fundó el movimiento de la Secesión, liderando a un grupo de jóvenes artistas que estaban fuera de los consagrados. En su pintura de 1899, Nuda Veritas, Klimt incluyó la cita del escritor alemán Friedrich Schiller (1759-1805): "Si no puedes complacer a todo el mundo con tus hazañas y tu arte, complace a unos pocos. Complacer a muchos es malo". Así anunciaba que complacer a sus clientes ya no era su prioridad ${ }^{3,4}$.

El tema del techo del Aula Magna de la Universidad era "el triunfo de la luz sobre la oscuridad". Consistía en un panel central rodeado por cuatro pinturas que representaban a las Facultades de la Universidad. El concepto fue bastante convencional, similar a otros realizados en los edificios públicos de toda Europa. Klimt tuvo en este encargo una oportunidad para desarrollar sus ideas revolucionarias sobre el arte y lo irracional. Pintó los cuadros que representaban a la Filosofía, Medicina y Jurisprudencia, mientras Franz Matsch emprendió Teología y el panel central Triunfo de la luz sobre la oscuridad ${ }^{1}$.

En mayo de 1898 presentaron los bocetos a una Comisión del Ministerio. Las críticas fueron dirigidas a Klimt y ambos artistas se vieron obligados a declararse "dentro de los límites de la libertad artística" para llevar a cabo las modificaciones necesarias para mantener una unidad estilística. "Filosofía" fue la primera de las tres obras presentadas al Gobierno austríaco durante la $7^{\circ}$ Exposición de la Secesión de Viena, en marzo de 1900, el mismo año en que Sigmund Freud (1856-1939) publicó, también en Viena, su obra "La interpretación de los sueños" 2 .

En "Filosofía" los espectadores se enfrentaron con una columna de figuras desnudas abrazándose contra un fondo de espacio ilimitado del cual emerge la cabeza soñolienta de una esfinge. El único signo de la mente consciente era una cabeza femenina, brillante, en la parte inferior del cuadro. Klimt describió el cuadro de la siguiente manera: "A la izquierda un grupo de figuras, el comienzo de la vida, el placer, la decadencia. A la derecha, el mundo como misterio. Abajo, una figura de luz emergiendo: el conocimiento". Se había separado de su anterior estilo historicista y había traído los nuevos elementos de la Secesión ${ }^{4}$.

La obra fue galardonada con una medalla de oro en la Exposición Universal de París, pero fue atacado por los de su propio país. Wilhelm von Neumann, rector de la universidad, afirmó que "en 
una época en que la filosofía buscaba la verdad en las ciencias exactas, aquélla no merecía ser representada como una construcción nebulosa y fantástica". Ochenta y siete profesores de la Universidad de Viena presentaron una petición ante el Ministerio solicitando que la pintura no ocupara su lugar predeterminado en la Universidad. Sólo un pequeño grupo de doce profesores universitarios apoyó públicamente el trabajo de Klimt, con una contra-petición. Dentro de esta minoría académica, el anatomista Emil Zuckerkandl (1848-1910) fue uno de los pocos que firmaron ${ }^{5}$. Klimt fue ratificado y continuó con el encargo, realizando en los años sucesivos "Medicina" y "Jurisprudencia".

\section{La medicina: análisis del cuadro}

A pesar de los ataques recibidos, Klimt presentó su segunda obra, "Medicina", (Figura 2) en marzo de 1901, durante la $10^{\circ}$ Exposición de la Secesión. Se trataba de un óleo sobre lienzo de 430 x $300 \mathrm{~cm}$. "Medicina" se parecía a "Filosofía", volviendo al entrelazamiento de cuerpos flotantes -el curso de la vida- en medio de los cuales aparece el esque-

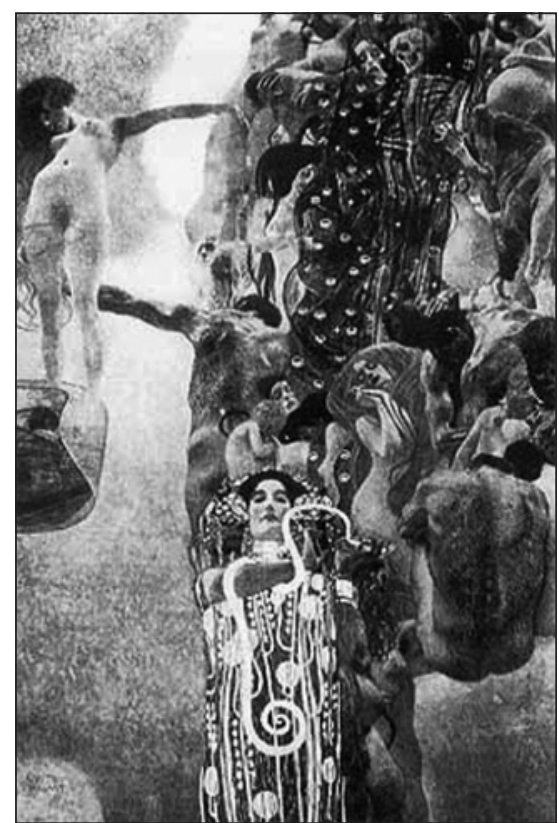

Figura 2. "Medicina": olio sobre tela 430 x $300 \mathrm{~cm}$. En: http://lacomunidad.elpais.com/psicoanalisis/2009/1/19/ entrevista-melia-diez-sobre-psicoanalisis-del-programa-tv; consultado 10/3/2013. leto de la muerte; una figura femenina se destaca de la columna, para representar la liberación del dolor. Ambos lienzos se quemaron y sólo fueron fotografiados en blanco y negro, (con excepción de Hygeia, Hygieia o Higea, de la que queda un detalle en color) (Figura 3). Hay constancia escrita de que, mientras "Filosofía" se basaba en tonos fríos, verdes y azules, en el nuevo panel los colores iban del rosa al púrpura.

La composición está presidida por Higea, que dio origen a la palabra "higiene", diosa de la salud e hija de Esculapio, ataviada con una larga túnica y con una larga serpiente enroscada en su brazo derecho que bebe de su copa, en su mano izquierda. Mira de frente al espectador, como si nos obligara a reconocer la visión existencial que aparece a sus espaldas con adornos circulares, rodeadas de sensuales mujeres desnudas, ancianos, hombre y niños, destacando la mujer embarazada (antecedente directo de otra obra de Klimt, "La esperanza”) que se sitúa junto a la Muerte. La humanidad está perdida en el espacio. Klimt recurrió una vez más al sufrimiento humano para reseñar la separación entre la ciencia y la humanidad.

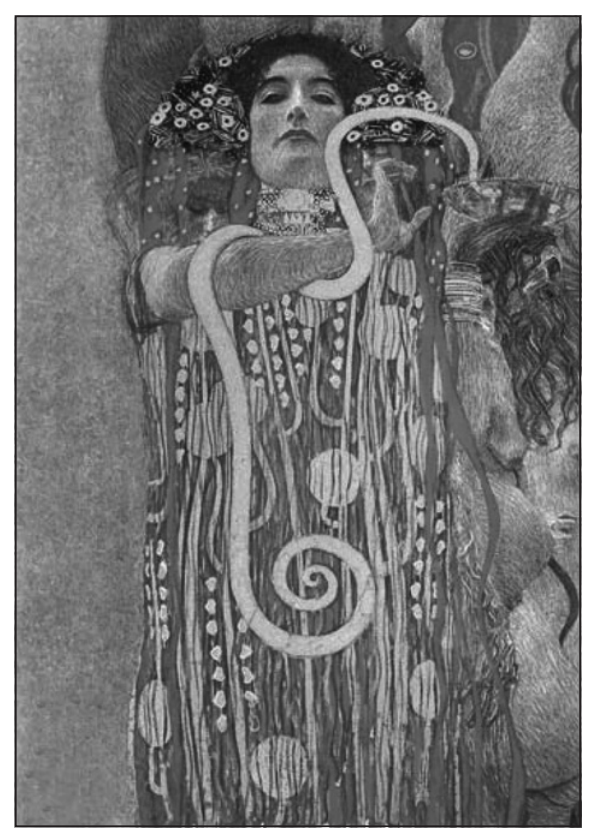

Figura 3. Gustav Klimt - Higea (Detalle de "Medicina"). En: http://psicoanalisisalud.blogspot.com.ar/2009_02_01_archive.html; consultado 10/3/2013. 
Transmitió una unidad ambigua de la vida y la muerte, sin nada que celebre el rol de la medicina o de la ciencia de curar. Era obvio que el pintor quiso resaltar la impotencia de las artes curativas y no hizo ningún intento por representar el triunfo de la medicina en la manera que los médicos esperaban. Cabe señalar que Klimt había sido testigo de la muerte de su padre y de su hermano, y vio morir a su hermana menor a la edad de 5 años. Su hermana mayor y su madre poco a poco perdieron su cordura. En una época en la que Viena era líder mundial en medicina, coincidiendo con la segunda Escuela de Viena que tuvo entre otros exponentes a von Rokitansky, Skoda, Kahler, Nothnagel, Billroth, Chvostek y Türk, entre otros. Estos grandes nombres, acompañados de descripciones formales clínicas, fisiopatológicas y las primeras medidas preventivas, llevaban a los médicos y estudiantes de la universidad a creer en la omnipotencia de las ciencias médicas. Reinaba la sensación de triunfo, la medicina finalmente vencería las enfermedades. El médico se sentía poderoso, imbatible. Frente a esto surge el cuadro de Klimt que funciona como recordatorio permanente de las limitaciones de la medicina. Los cuerpos amontonados, sufrientes, la presencia fuerte y central de la muerte, que lejos de ser vencida parece levantarse del dolor. La muerte seguía derrotando a la medicina, el caos triunfaba sobre la racionalidad. Claramente no era eso lo que los médicos esperaban ver, el cuadro no representaba el triunfo de la prevención y la cura, sino que ponía de manifiesto las limitaciones de la medicina frente a la fuerza de la enfermedad y la muerte. Por otro lado, Higea -si bien es una figura central- parece alejada, como testigo involuntaria del caos reinante.

A la izquierda del cuadro, la figura de una mujer joven, sensual, con un niño a sus pies que representa la vida que se erige ante tanta muerte, el río de la vida que no se detiene y garantiza la continuidad de la especie.

Un editorial en el Medizinische Wochenschrift se quejó de que el pintor había ignorado los dos logros principales de los médicos: la prevención y la curación. El periodista más mordaz de Viena, Karl Krauss (1874-1936), describió "Medicina" como una pintura en que la confusión caótica de cuerpos decrépitos simboliza la situación de un hospital estatal.

¿Quién puede culpar a Klimt por la falsa om- nipotencia de los médicos, por ser un recordatorio gráfico e imponente de nuestras limitaciones?

Las fuertes críticas recibidas y la polémica creada alrededor del lienzo provocaron la reacción de Klimt al pintar el tercer cuadro de la serie: "Jurisprudencia".

"Jurisprudencia" fue presentada en la $18^{\text {a }} \mathrm{Ex}-$ posición de la Secesión, en 1903. Aquí, el maestro austríaco plasmó toda su ira y su indignación. La obra definitiva cambia radicalmente respecto al boceto preparatorio, e incluso a sus compañeras "Filosofía" y "Medicina". La figura central es una víctima indefensa de la ley, no un símbolo de la Justicia.

Las tres pinturas fueron atacadas por los críticos, ya que cada una rompía tabúes culturales diferentes, lo que contradecía la tendencia de la época de "sublimar la realidad y presentar solamente sus aspectos más favorables". Las pinturas sumaban a los cargos de obscenidad que a menudo enfrentaban a Klimt. Intervino un fiscal y el tema llegó hasta el Parlamento de Austria. Fue la primera vez que un debate cultural alcanzó esa instancia. Pero, finalmente no se tomó ninguna medida. Sólo el Ministro de Educación Wilhelm von Hartel defendió a Klimt, pero cuando el artista fue elegido para ser Profesor en la Academia de Bellas Artes, en 1901, el gobierno se negó a ratificar su puesto. Nunca más se le ofreció otro puesto de profesor. Esta también sería la última vez que Klimt aceptara comisiones por parte del Estado, señalando: "Ya he tenido suficiente de la censura. Rechazo todo el apoyo estatal, no quiero nada de eso".

Una pintura de Klimt titulada "Goldfish", que más tarde re-tituló "A mis críticos" (1901-1902), fue presentada en la 13a Exhibición de la Secesión, en Dresden, en 1904. Esta obra muestra a una hermosa mujer sonriente proyectando su trasero al espectador, y es una obvia respuesta a todas las personas que atacaron la "pornografía" y el "exceso de perversidad" de las pinturas de la Universidad.

La Comisión de Arte de la Universidad dictaminó en 1903 que los tres lienzos pintados por Klimt debían instalarse en la Galería de Arte Moderno de Viena. Franz Matsch sería el encargado de completar el programa iconográfico, ya que Klimt pronto se retiraría del proyecto. Las pinturas fueron solicitadas para la Exposición de Compras de Luisiana, en 1904, en St. Louis, Missouri, pero el Ministerio se negó, temeroso de la reacción que generaría. Klimt luego renunció al 
encargo, con el deseo de mantener sus obras, pero el Ministerio insistió en que ya eran propiedad del Estado. Klimt debió amenazar con una escopeta al personal que fue a retirar las obras de su casa, para poder quedárselas. Devolvió su adelanto de 30.000 coronas con el apoyo de August Lederer, uno de sus clientes más importantes, que a cambio de ello recibió "Filosofía". En 1911, "Medicina” y "Jurisprudencia" fueron compradas por su amigo y colega, el artista Koloman Moser. Poco después, los tres cuadros de la facultad encontraron su lugar en la Galería Austríaca, pero en 1938 las pinturas fueron capturadas por los alemanes.

En 1943, después de una exposición final, fueron trasladadas a Immendorf Schloss, un castillo en la Baja Austria, para su protección. En mayo de 1945 las pinturas fueron destruidas (con otras 14 obras de Klimt y otros autores) cuando las fuerzas alemanas de la SS en retirada prendieron fuego al castillo para evitar que cayera en manos enemigas (otra versión dice que el incendio lo provocaron las tropas rusas cuando tomaron el castillo). Queda sólo una fotografía de la pintura completa de "Medicina", tomada justo antes de que fuera destruida. También han quedado bocetos preparatorios, entre ellos uno al óleo de "Medicina" y una foto a color de Higea (Figura 3).

$\mathrm{Su}$ "Filosofía" muestra una inspiración proporcionada por las ideas del compositor Richard Wagner (1813-1383) y el filósofo Friedrich Nietzsche (1844-1900), e Higea revela la conciencia del mundo simbólico de la mitología. Gustav Klimt y Sigmund Freud compartían la misma ciudad y el mismo entorno cultural. Comparando las intenciones del artista con el enfoque del psicoanalista se ha propuesto una "crisis del ego liberal". Ambos pasaron por una crisis personal, reformulando su trabajo y enfrentando el rechazo cuando exhibieron los resultados de sus exploraciones en el caótico mundo de los instintos.

Klimt tuvo su época dorada, determinada por un progresivo acercamiento de la crítica y un gran éxito comercial. Muchas de sus pinturas en este período incorporan pan de oro. Las obras más notables realizadas en esta etapa fueron el "Retrato de Adele Bloch-Bauer I" (1907) y "El beso" (1907-1908) ${ }^{1}$.

En 1911, gracias a "La vida y la muerte" (Figura 4), cuadro relacionado a la medicina, es galardonado con el primer premio de la Exposición Uni-

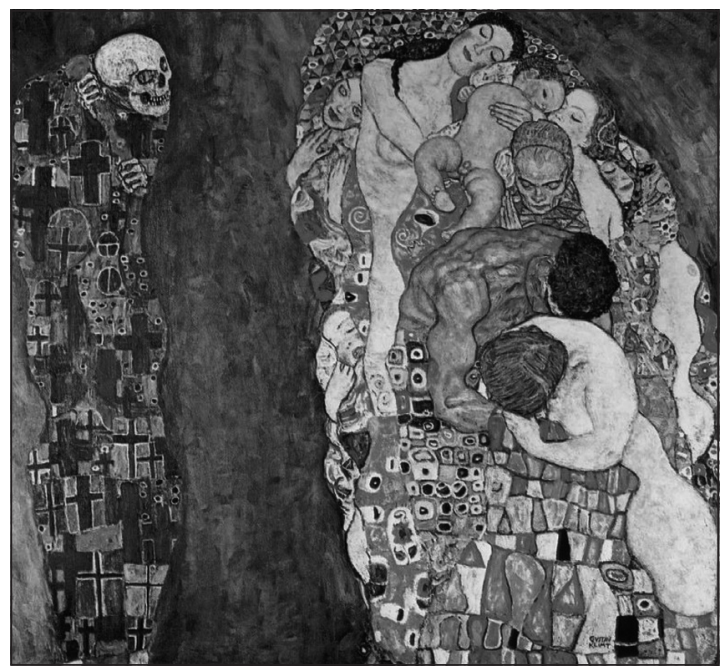

Figura 4. "Muerte y Vida" (1916). En: http://criaturasimaginarias.wordpress.com/2007/08/15/muerte-y-vida-gustavklimt; consultado 10/3/2013.

versal de Roma. En 1915, murió su madre, Anna. Tres años más tarde, a los 55 años y luego de haber sufrido un accidente cerebrovascular, y la llamada gripe española, falleció el pintor.

Klimt es universalmente reconocido como un pionero del arte moderno vienés, y las pinturas de la Facultad son consideradas como un punto de inflexión en su brillante carrera.

\section{Referencias}

1. Néret G. Gustav Klimt: 1862-1918. Los Angeles, Estados Unidos. Editorial Taschen; 2007. p. 7-91.

2. Colimi A. Gustav Klimt. New York, Estados Unidos. Editorial Geoge Braziller; 1973. p. 5-31.

3. Bitsori M, Galanakis E. Doctors versus artists: Gustav Klimt's Medicine. BMJ 2002; 325: 1506-8.

4. Plata Rosas LJ. Gustav Klimt: una dosis de amarga medicina. En: Un científico en el museo de arte moderno: encuentros cercanos entre el arte y la ciencia. Buenos Aires, Argentina: Editorial siglo veintiuno; 10 Ed; 2011. p. 105-8.

5. Schultheiss D. Gustav Klimt and the field of medicine. Painting of the medical faculty-relationship with the Zuckerkandl family. Urology 2007; 46: 1005-10.

6. Young P, Finn BC, Bruetman JE, Cesaro Gelos J, Trimarchi H. La vara de Esculapio, símbolo de la medicina. Rev Med Chile 2013; 141: 1208-12. 